01,07

\title{
Зернограничное проскальзывание и миграция специальных границ зерен в бикристаллах Al. Атомистическое моделирование
}

\author{
(C) Л.Е. Карькина ${ }^{1}$, И.Н. Карькин ${ }^{1}$, А.Р. Кузнецов ${ }^{1,3}$, Ю.Н. Горностырев ${ }^{1,2}$ \\ ${ }^{1}$ Институт фозики металлов УрО РАН, \\ Екатеринбург, Россия \\ ${ }^{2}$ Институт квантового материаловедения, \\ Екатеринбург, Россия \\ ${ }^{3}$ Уральский федеральный университет, \\ Екатеринбург, Россия \\ E-mail: Lidiya.karkina@imp.uran.ru
}

(Поступила в Редакцию 10 апреля 2018 г.

В окончательной редакции 25 апреля 2018 г.)

Для выяснения механизмов, контролирующих процессы проскальзывания и миграции границ зерен (ГЗ), энергетика зернограничных сдвигов для нескольких типичных симметричных ГЗ наклона $\Sigma 3$ (111), $\Sigma 5(012), \Sigma 5(013)$ и $\Sigma 11$ (113) в бикристалле Al рассчитана с использованием методов ab initio и молекулярно-динамического моделирования. Определена энергия обобщенных зернограничных дефектов упаковки $(\mathrm{GB}-\mathrm{SF})$, установлены предпочтительные направления и энергетический барьер для зернограничного проскальзывания. Показано, что относительное смещение соседних зерен при определенных направлениях частичных сдвигов сопровождается консервативной миграцией ГЗ в направлении, перпендикулярном плоскости ее плоскости. Результаты моделирования сопоставляются с известными механизмами зернограничного скольжения Al.

Работа выполнена в рамках государственного задания по темам „Давление“ № AАAА-А18-118020190104-3 и „Структура“ № AААА-А18-118020190116-6.

DOI: 10.21883/FTT.2018.10.46511.099

\section{1. Введение}

Проскальзывание по границам зерен (ГЗ) и миграции ГЗ являются процессами, которые, наряду с внутризеренным скольжением и процессами аккомодации в тройных стыках, обеспечивают пластическую деформацию поликристаллических материалов. Явление инициированной напряжением миграции ГЗ, впервые изученное на бикристаллах $[1,2]$, в последнее время привлекает повышенный интерес и рассматривается как альтернативный механизм пластичности нанозеренных материалов, в которых внутризеренное скольжение затруднено или подавлено [3-11]. Исследование этого явления вызвало ряд новых вопросов относительно основных механизмов, определяющих миграцию ГЗ, а также взаимосвязи процессов миграции и зернограничного проскальзывания (shear-migration coupling).

К настоящему времени существует большое количество экспериментальных и теоретических исследований, посвященных изучению механизмов миграции и проскальзывания ГЗ (см. работы [8,11-15] и ссылки в них). Рассматриваемые процессы в общем случае являются достаточно сложными и зависят от особенностей структуры Г3 [11], развития аккомодационных процессов в стыках зерен, которые, в свою очередь, могут протекать с участием диффузионного массопереноса [10].

В последнее время внимание привлекают „консервативные“ механизмы миграции и проскальзывания, способные обеспечивать зернограничную пластичность при комнатной и более низких температурах, когда внутризеренная диффузия заморожена. Обсуждаемые модели этих процессов $[8,13,14]$ включают лишь локальные перестановочные смещения атомов (localized atomic shuffling) и не учитывают диффузионный массоперенос на большие расстояния. Известным примером консервативного движения ГЗ, не связанного с какой-либо диффузионной перетасовкой атомов является движение двойниковых границ. Отметим, что нанодвойники играют большую роль в процессах пластической деформации нанозерен в ГЦК-материалах с низким значением энергии дефекта упаковки (stacking fault - SF) [16-18].

Однако предложенные модели взаимосвязанной миграции и проскальзывания ГЗ являются, главным образом, геометрическими $[8,13,14]$, и не делают различия между процессами на малоугловых и на большеугловых границах зерен. В частности, для большеугловых ГЗ предложена [8] модель коррелированного поворота и смещения малого объема вблизи ГЗ. Такая деформация обеспечивает миграцию ГЗ на величину $m$ и смещение вдоль ГЗ на величину $s$. Параметр связи $\beta=s / m$ зависит от типа ГЗ, величины приложенного сдвигового напряжения и температуры, изменяясь в пределах от 0 до 1.

Для развития последовательных представлений о процессах, контролирующих миграцию и проскальзывание ГЗ необходим переход на микроскопический уровень описания, учитывающий структуру ГЗ и особенности межатомных взаимодействий в конкретных материалах. 
В настоящей работе методами атомистического моделирования, мы анализируем энергетические характеристики процесса проскальзывания для ряда симметричных границ зерен специального типа $(\Sigma 5\{012\}, \Sigma 5\{013\}$, $\Sigma 11\{113\}, \Sigma 3\{111\})$ в Al. Полученные результаты позволили определить предпочтительные направления векторов зернограничных сдвигов, и выяснить условия, при которых проскальзывание может сопровождаться миграцией границы.

\section{2. Метод расчета}

Чтобы охарактеризовать присущее данному типу ГЗ сопротивление зернограничному сдвигу мы провели расчет энергии обобщенного дефекта упаковки (GB-SF) или зернограничной $\gamma$-поверхности для плоскости границы, разделяющей два зерна. Величина обобщенного ДУ часто используется при обсуждении расщепления дислокаций и оценке сопротивления скольжению $[19,20]$; в этом случае она характеризует изменение энергии при сдвиге частей кристалла в плоскости скольжения дислокаций. Энергия GB-SF рассчитывалась для специальных границ зерен в ГЦК Al с осями наклона $\langle 001\rangle$ и $\langle 110\rangle$. Были рассмотрены симметричные низкоэнергетические ГЗ [21]: $\Sigma 5\{012\}\langle 100\rangle, \Sigma 5\{013\}\langle 100\rangle$ и $\Sigma 11\{113\}\langle 110\rangle$, $\Sigma 3\{111\}\langle 110\rangle$.

Расчет энергетической поверхности GB-SF проводился методом молекулярной динамики (МД) с использованием пакета LAMMPS [22]; с целью уточнения полученных результатов для отдельных направлений сдвига проводился $a b$ initio расчет методами теории функционала электронной плотности. При МД-моделировании для $\mathrm{Al}$ использовался adp-потенциал межатомного взаимодействия, предложенный в работе [23] (Pot1), а также EAM/FS потенциал [24] (Pot2). Эти потенциалы наилучшим образом описывают свойства алюминия и чаще всего используются в МД-расчетах [25].

$A b$ initio расчеты полной энергии кристаллита с ГЗ проводились в рамках теории функционала плотности с использованием пакета VASP (Vienna Ab-initio Simulation Package [26,27]) с псевдопотенциалами, построенными с помощью метода проекционных присоединенных волн (PAW) [28]. Обменно-корреляционная энергия находилась с использованием обобщённого градиентного приближения (GGA), формализм которого изложен в $[29,30]$. Энергия обрезания волновых функций в PAW расчетах составляла $520 \mathrm{eV}$. Интегрирование по зоне Бриллюэна проводилось с использованием $2 \times 4 \times 8$ сетки $k$-точек. Критерий сходимости по полной энергии составлял $10^{-5} \mathrm{eV} /$ cell. Расчетная часть работы была выполнена с использованием оборудования центра коллективного пользования „Комплекс моделирования и обработки данных исследовательских установок мегакласса“ НИЦ „Курчатовский институт“, http://ckp.nrcki.ru/.

Во всех случаях использовался кристаллит, плоскость $Y Z$ которого совпадала с плоскостью ГЗ, ось $O Z-$ параллельна оси наклона, направление $O X$ перпендикулярно плоскости границы. Кристаллит содержал два
Таблица 1. Размеры кристаллитов для изученных границ зерен (в единицах параметра решетки $a$ )

\begin{tabular}{c|c|c|c}
\hline \multirow{2}{*}{ Границы зерен } & \multicolumn{3}{|c}{ Вдоль направления } \\
\cline { 2 - 4 } & $\mathrm{OZ}$ & OY & OX \\
\hline$\Sigma 5\{012\}\langle 100\rangle$ & $6\langle 100\rangle$ & $2\langle 012\rangle$ & $20\langle 021\rangle$ \\
$\Sigma 5\{013\}\langle 100\rangle$ & $6\langle 100\rangle$ & $2\langle 013\rangle$ & $16\langle 031\rangle$ \\
$\Sigma 11\{113\}\langle 110\rangle$ & $5\langle 110\rangle$ & $\langle 332\rangle$ & $16\langle 113\rangle$ \\
$\Sigma 3\{111\}\langle 110\rangle$ & $5\langle 110\rangle$ & $3\langle 112\rangle$ & $16\langle 111\rangle$
\end{tabular}

зерна, разделенные рассматриваемой ГЗ, расположенной в его центре. При МД-моделировании кристаллит содержал в зависимости от типа ГЗ от 40000 до 80000 атомов; размеры кристаллитов приведены в табл. 1 . Во всех случаях размер зерен вдоль направления $O X$, перпендикулярного плоскости ГЗ составлял $\sim 10 \mathrm{~nm}$. В этом направлении использовались свободные граничные условия. В двух других взаимно перпендикулярных направлениях, лежащих в плоскости ГЗ, использовались периодические граничные условия. Длина периода вдоль этих направлений была кратна трансляции вдоль заданного кристаллографического направления $(a, \sqrt{5} a$ и $1 / 2 \sqrt{10} a$ вдоль направлений $\langle 100\rangle,\langle 012\rangle$ и $\langle 013\rangle$ соответственно). Размер кристаллита, используемого для $a b$ initio расчетов, составлял 40-90 атомов; во всех направлениях использовались периодические граничные условия.

Во все рассмотренных случаях минимуму энергии соответствует структура ГЗ, симметричная относительно их плоскости, а относительное смещение зерен приводит к повышению энергии (см. раздел 3). При расчете $\mathrm{GB}-\mathrm{SF}$ сдвиг одного зерна относительно другого проводился по плоскости, ближайшей к геометрическому центру ГЗ. Величина GB-SF вычислялась как изменение энергии кристаллита (отнесенное к площади границы) при сдвиге на заданный вектор f, лежащий в пределах элементарной ячейки решетки совпадающих узлов (РСУ) в плоскости ГЗ. После осуществления сдвига в точках локальных минимумов GB-SF проводилась полная релаксация кристаллита. В других точках, не отвечающему локальному минимуму (точки общего положения), проводилась релаксация только в направлении $O X$, перпендикулярном плоскости ГЗ. Полученные таким образом значения $E_{\mathrm{GB}-\mathrm{SF}}(\mathbf{f})$ образуют $\gamma$-поверхность, анализ которой позволяет сделать выводы о существовании стабильных поверхностных дефектов упаковки и определить сопротивление сдвигу вдоль того или иного направления.

\section{3. Результаты расчетов энергии зернограничных дефектов упаковки}

3.1. Г3 с осью наклона $\langle\mathbf{1 0 0}\rangle$. На рис. 1 представлены нерелаксированные зернограничные $\gamma$-поверхности для изученных границ зерен с осью наклона $\langle 100\rangle$. 

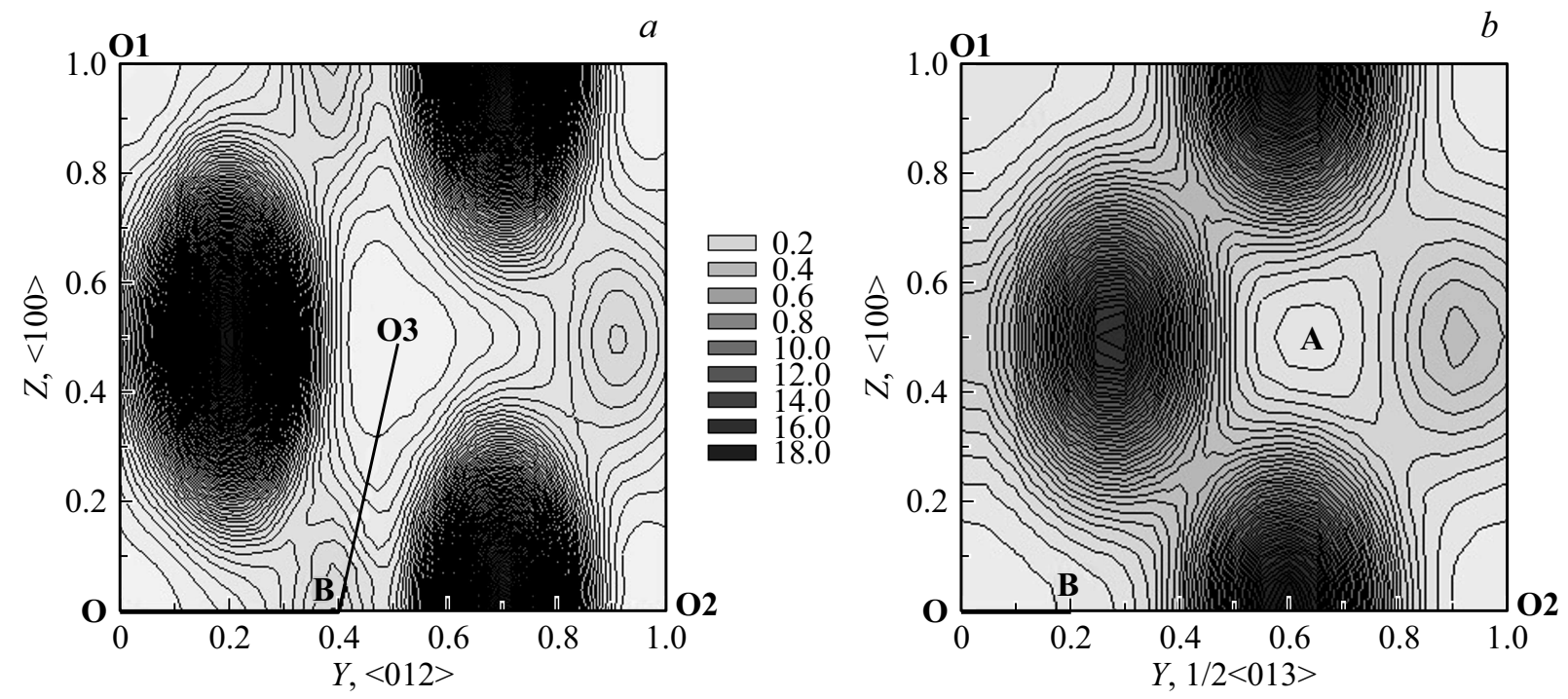

Рис. 1. Поверхности GB-SF, рассчитанные с использованием потенциала Pot1 для $(a)-\Gamma 3 \Sigma 5\{012\}\langle 100\rangle,(b)-\Sigma 5\{013\}\langle 100\rangle$. Значения энергий даны в $\mathrm{J} / \mathrm{m}^{2}$
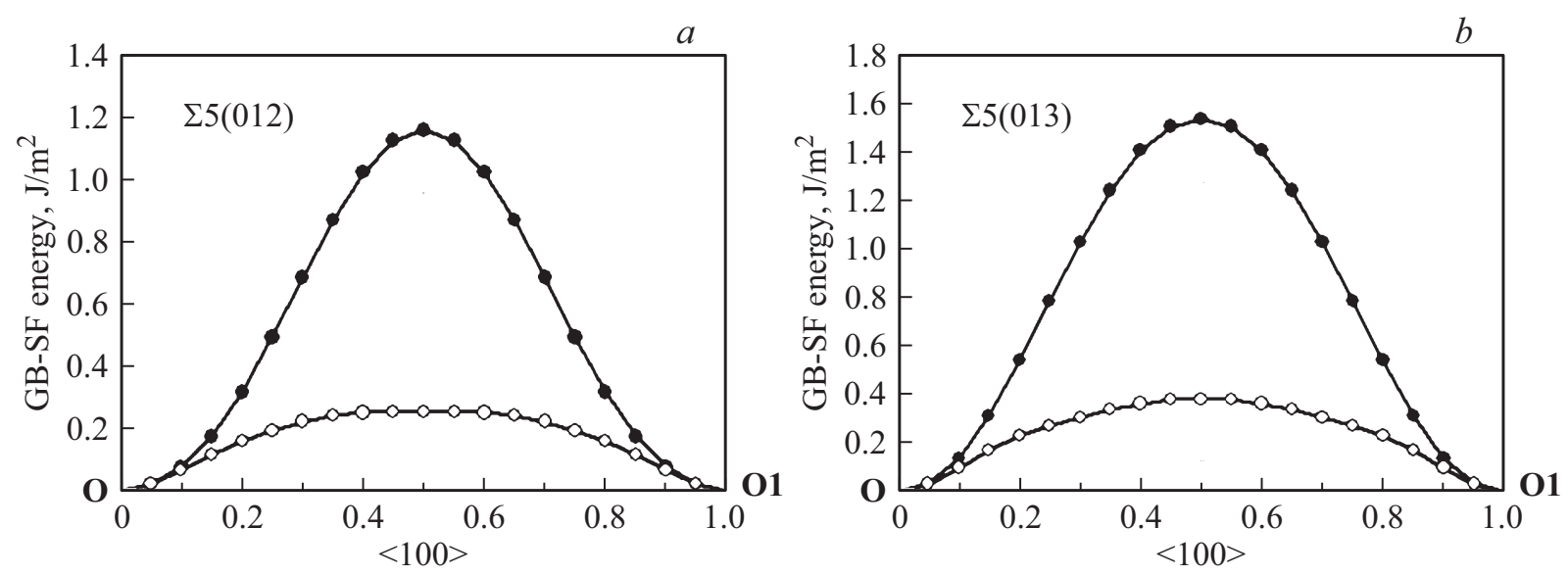

Рис. 2. Зависимость энергии GB-SF (потенциал Pot1) вдоль направления $\langle 100\rangle$ для границ $\Sigma 5\{012\}(a)$ и $\Sigma 5\{013\}(b)$. Темные точки - без релаксации, Светлые точки - с учетом релаксации.

Во всех случаях вдоль направлений параллельных и перпендикулярных оси наклона в плоскости границы длины даны в относительных единицах, по отношению к трансляции вдоль соответствующего кристаллографического направления. Векторы О01, О02, 003 отвечают трансляциям в элементарных ячейках РСУ. Для ГЗ $\Sigma 5\{013\}\langle 100\rangle$ на $\gamma$-поверхности присутствует дополнительный минимум в точке А (рис. $1, b)$ с координатами $(0.65 ; 0.5)$. Значение энергии SF в точке A без релаксации равно $0.65 \mathrm{~J} / \mathrm{m}^{2}$ и равно нулю с учетом релаксации.

Как видно из рис. 1, одним из легких направлений сдвига для всех рассмотренных границ является направление О01, параллельное оси наклона $\langle 100\rangle$. Кроме того, возможными направлениями сдвига являются ОО3 (для ГЗ $\Sigma 5\{012\}\langle 100\rangle$ ), ОА и О2А (для ГЗ $\Sigma 5\{013\}\langle 100\rangle)$. На рис. 2 представлена энергия GB-SF в зависимости величины сдвига вдоль направления $\langle 100\rangle$.
Из сравнения кривых, представленных на рис. 2 видно, что для всех рассмотренных ГЗ релаксация приводит к существенному понижению энергии. В точке максимума, соответствующей сдвигу $\mathbf{f}=1 / 2\langle 100\rangle$, значение энергии зернограничного дефекта упаковки понижается в 3.5-4.5 раза; однако, атомные смещения вблизи плоскости границы при релаксации не превосходят 1\%. Максимальное значение энергии GB-SF вдоль выбранного направления сдвига соответствует нестабильному дефекту упаковки, величина которого $\gamma_{\text {us }}$ характеризует сопротивление решетки при зернограничном скольжении. Ранее величина $\gamma_{\text {us }}$ была введена в теории дислокаций $[19,31]$, где она определяет энергетический барьер, который необходимо преодолеть для распространения сдвига. В табл. 2 представлены значения энергии $\gamma_{\text {us }}$ вдоль направления $O Y$ для двух потенциалов (Pot1 и Pot2) в сравнении с результатами $a b$ initio расчетов. 

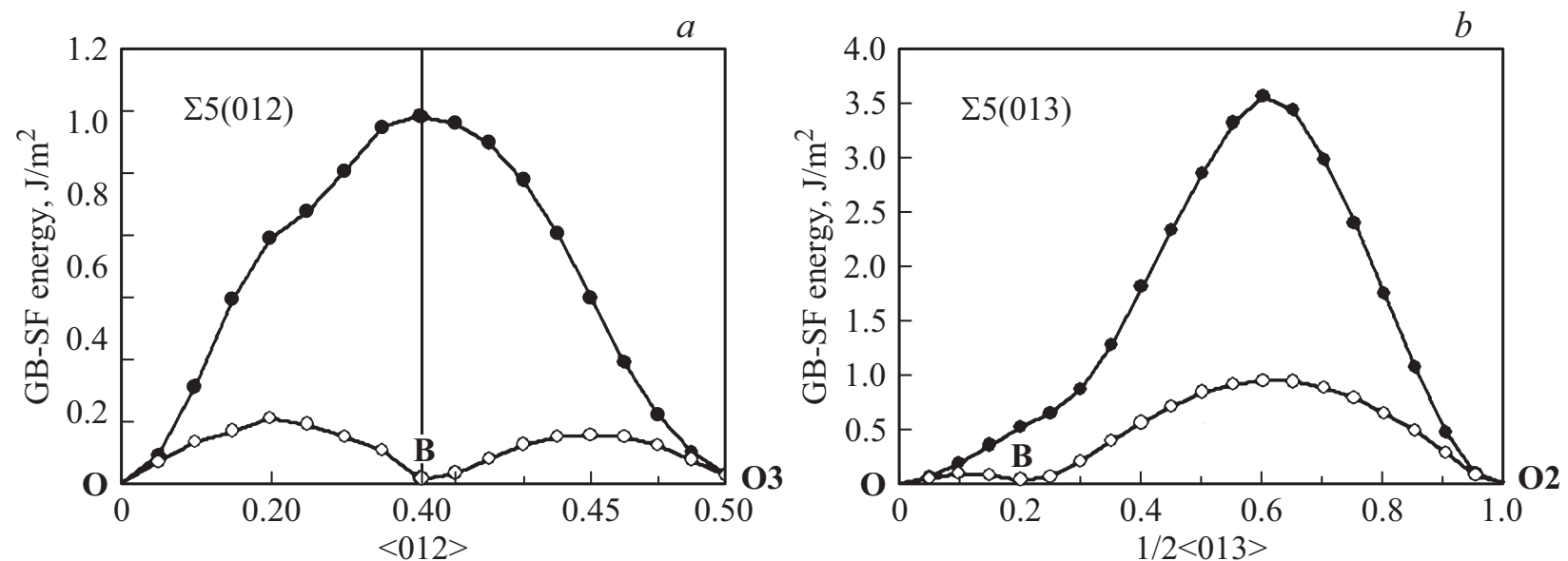

Рис. 3. Изменение энергии GB-GSF (потенциал Pot1) при сдвиге вдоль пути ОВ-ВО3 для $\Sigma 5\{012\}(a)$ и вдоль пути ОВ-ВО2 для $\Sigma 5\{013\}(b)$. Темные символы - без релаксации, светлые - с учетом релаксации.

Из табл. 2 видно, что для всех границ релаксация значительно (почти на порядок) понижает значение $\gamma_{\text {us. }}$. Релаксированные значения $\gamma_{\text {us }}$ для обоих потенциалов Pot1 и Pot2 оказались близки и согласуются с результатами ab initio расчетов. Сопротивление сдвигу вдоль $\langle 100\rangle$ для ГЗ $\Sigma 5\{012\}$ примерно в 1.5 раза ниже, чем для ГЗ $\Sigma 5\{013\}$.

Реализация сдвигов в направлениях 003 (для ГЗ $\Sigma 5\{012\}\langle 100\rangle$ ) и ОА (для ГЗ $\Sigma 5\{013\}\langle 100\rangle$ ) требует преодоления значительных барьеров (см. рис. 1). Более легким является осуществление сдвига вдоль составного пути, с участием частичного сдвига вдоль легкого направления, перпендикулярного оси наклона $(\langle 012\rangle$ в случае $\Sigma 5\{012\}\langle 100\rangle$ и $\langle 013\rangle$ в случае $\Sigma 5\{013\}\langle 100\rangle)$. Изменение энергии GB-SF при реализации таких сдвигов представлено на рис. 3. В результате релаксации вблизи точки В, соответствующей сдвигам $0.4(\langle 021\rangle)$ и $0.2(1 / 2\langle 013\rangle)$ (величина сдвига $0.9 a$ и $0.32 a$ соответственно), формируется локальный минимум, энергия которого близка к нулю. Это означает, при таком сдвиге реализуется координация атомов, близкая к начальной конфигурации ГЗ. Для границы $\Sigma 5\{012\}\langle 100\rangle$, после осуществления сдвига ОВ дальнейшее проскальзывание в том же направлении затруднено, так как приводит к образованию ДУ нагромождения с высокой энергией (рис. $3, a)$, но может происходить вдоль направления ВО3 (рис. 1) с относительно низкой величиной барьера. Для ГЗ $\Sigma 5\{013\}\langle 100\rangle$ дальнейшее относительное смещение зерен как вдоль направления ВО2, так и вдоль направления ВА затруднено, поскольку сопровождается значительным повышением энергии (рис. $3, b$ ).

Проскальзывание на вектор частичного сдвига ОВ будет сопровождаться перестройкой структуры ГЗ, вызывающей ее миграцию в новое положение. На рис. 4 показаны изменения структуры рассматриваемых границ в начальной конфигурации и после частичного сдвига ОВ с полной релаксацией; штриховая линия разделяет области кристалла, которые сдвигалась друг относи- тельно друга. Тонкими линиями на рисунках выделены структурные единицы, геометрия которых сохраняется после соответствующих сдвигов и полной релаксации. Из рис. 4 видно, что в результате сдвига плоскость ГЗ перемещается на величину, равную вектору Бюргерса зернограничных сдвигов $\mathbf{b}_{\mathrm{gb}}$ [32]. Для ГЗ $\Sigma 5\{012\}$ величина $\mathbf{b}_{\mathrm{gb}}(\langle 012\rangle)=1 / 10\langle 012\rangle \quad(\sim 0.22 \AA)$, а для ГЗ $\Sigma 5\{013\}$ плоскость границы перемещается на величину $2 \mathbf{b}_{\mathrm{gb}}(\langle 013\rangle) \sim 0.32 \AA$, где $\mathbf{b}_{\mathrm{gb}}(\langle 013\rangle)=1 / 20\langle 013\rangle$.

Зернограничное проскальзывание может быть продолжено путем осуществления частичного сдвига в следующей, параллельной ГЗ плоскости, соответствующей новому положению ГЗ после миграции. В результате многократного повторения частичного сдвига по последовательной системе параллельных плоскостей

Таблица 2. Энергии нестабильных $\mathrm{SF}\left(\gamma_{\text {us }}, \mathrm{J} / \mathrm{m}^{2}\right)$ для сдвига вдоль направления $\langle 100\rangle$

\begin{tabular}{c|c|c|c|c}
\hline $\begin{array}{c}\text { Метод } \\
\text { расчета }\end{array}$ & $\begin{array}{c}\Sigma 5\{012\} \\
\text { unrelax }\end{array}$ & $\begin{array}{c}\Sigma 5\{012\} \\
\text { relax }\end{array}$ & $\begin{array}{c}\Sigma 5\{013\} \\
\text { unrelax }\end{array}$ & $\begin{array}{c}\Sigma 5\{013\} \\
\text { relax }\end{array}$ \\
\hline Pot1 & 1.16 & 0.25 & 1.54 & 0.38 \\
Pot2 & 1.25 & 0.28 & 2.02 & 0.36 \\
ab initio & 1.74 & 0.25 & 2.58 & 0.37
\end{tabular}

Таблица 3. Энергии нестабильных $\mathrm{SF}\left(\gamma_{\text {us }}, \mathrm{J} / \mathrm{m}^{2}\right)$ для сдвига вдоль направления 0Y, перпендикулярного оси наклона границ $\Sigma 5\{012\}$ и $\Sigma 5\{013\}$

\begin{tabular}{c|c|c|c|c}
\hline $\begin{array}{c}\text { Метод } \\
\text { расчета }\end{array}$ & $\begin{array}{c}\Sigma 5\{012\} \\
\text { unrelax }\end{array}$ & $\begin{array}{c}\Sigma 5\{012\} \\
\text { relax }\end{array}$ & $\begin{array}{c}\Sigma 5\{013\} \\
\text { unrelax }\end{array}$ & $\begin{array}{c}\Sigma 5\{013\} \\
\text { relax }\end{array}$ \\
\hline Pot1 & 0.67 & 0.18 & 0.19 & 0.09 \\
Pot2 & 1.02 & 0.13 & 0.14 & 0.04 \\
ab initio & 0.87 & 0.20 & 0.17 & 0.05
\end{tabular}



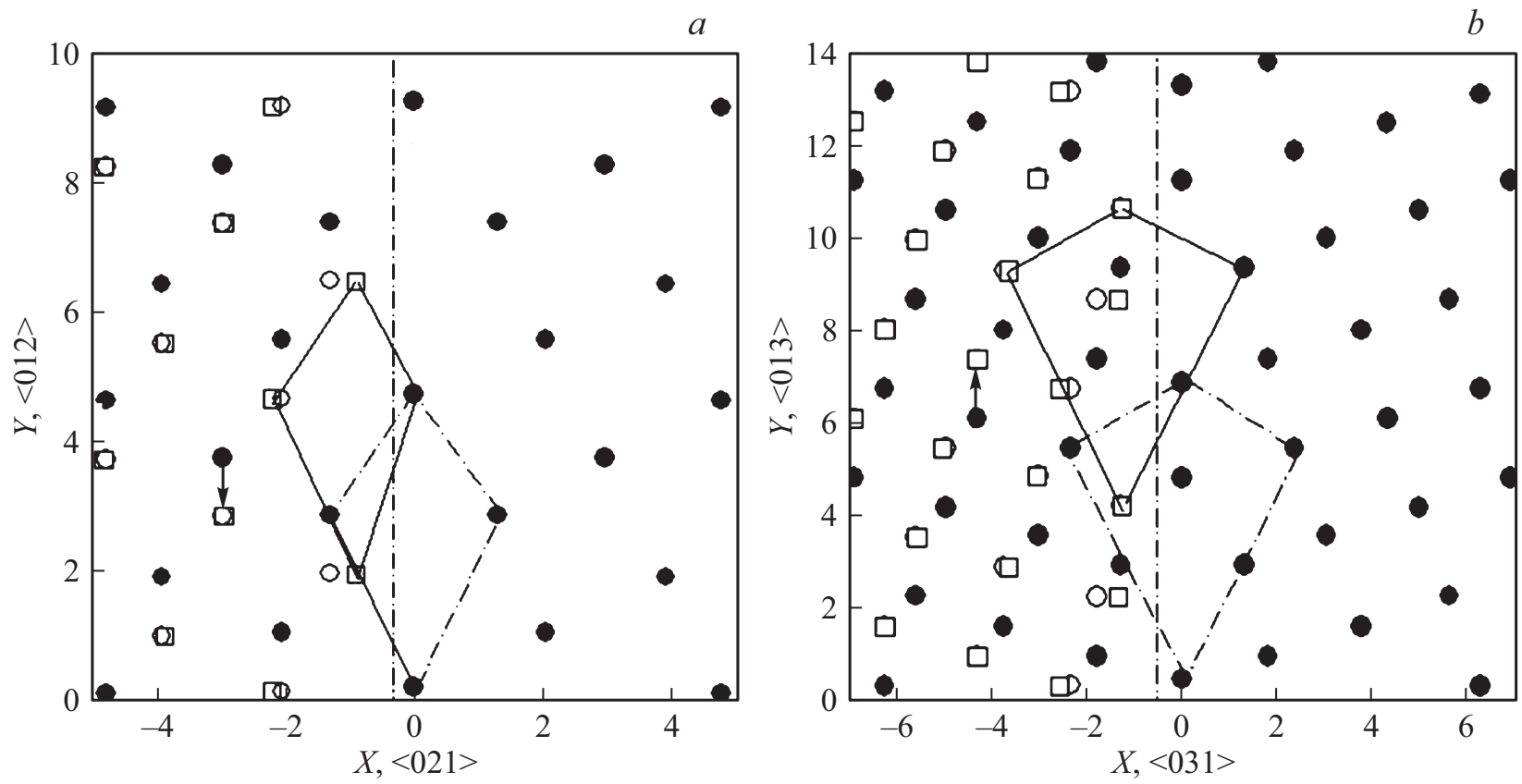

Рис. 4. Изменение структуры ГЗ $\Sigma 5\{012\}$ (a) и ГЗ $\Sigma 5\{013\}$ (b) при сдвиге на вектор ОВ. Темные точки - начальная конфигурация; окружности - сдвиг без релаксации; квадраты - с учетом релаксации после сдвига. Штрих-пунктирная линия структурная единица в начальном положении; сплошные линии - структурная единица после сдвига и релаксации.
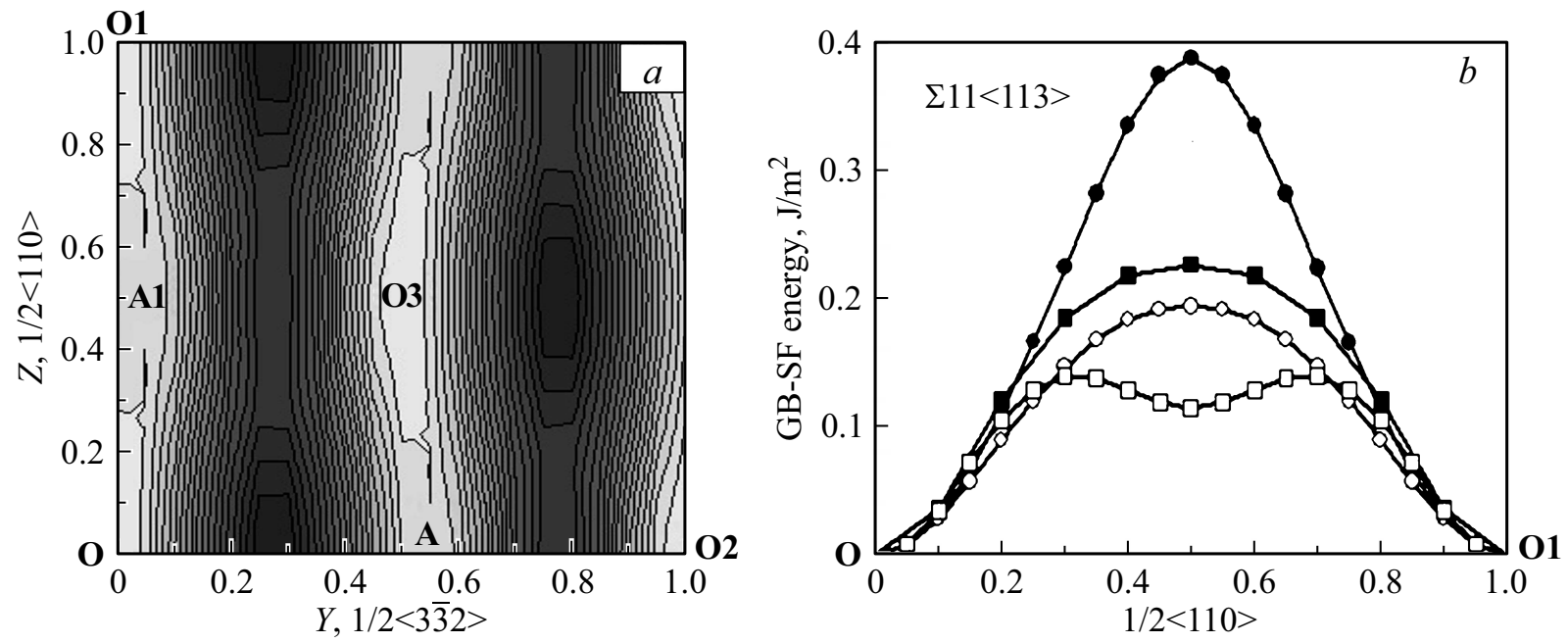

Рис. 5. GB-SF поверхность $(a)$ для потенциала Pot1 и зависимость энергии GB-SF вдоль направления $\langle 110\rangle(b)$ для ГЗ $\Sigma 11\{113\}\langle 110\rangle$. Точки на кривых отвечают потенциалу Pot1, квадраты - Pot2. Темные символы - без релаксации, светлые - с учетом релаксации.

реализуемый процесс зернограничного проскальзывания оказывается связан с миграцией ГЗ.

В табл. 3 представлены энергии нестабильных SF, характеризующие сопротивление сдвигу зерен вдоль легкого направления ОВ, перпендикулярного оси наклона ГЗ. Видно, что значения $\gamma_{\text {us }}$ в этом случае существенно ниже, чем в направлении 001, параллельном оси наклона (табл. 2). Сдвиг вдоль направления О2А (рис. 1, $b$ ) для ГЗ $\Sigma 5\{013\}$ затруднен, так как значения $\gamma_{\mathrm{us}}=0.51$ и $0.40\left(\mathrm{~J} / \mathrm{m}^{2}\right)$ с учетом релаксации для потенциалов Pot1 и Pot 2 соответственно значительно превышают значения энергий $\gamma_{\text {us }}$ для других рассмотренных выше сдвигов в этой плоскости.

3.2. ГЗ с осью наклона $\langle\mathbf{1 1 0}\rangle$. На рис. 5 представлена энергетическая поверхность GB-SF (без релаксации) для ГЗ $\Sigma 11\{113\}\langle 110\rangle$ и ее сечение вдоль направления $\langle 110\rangle$ без релаксации и с учетом релаксации вдоль направления, перпендикулярного плоскости ГЗ. Векторы 001, О02, 003 соответствуют трансляциям на элементарной ячейке РСУ данной границы. Видно, что 

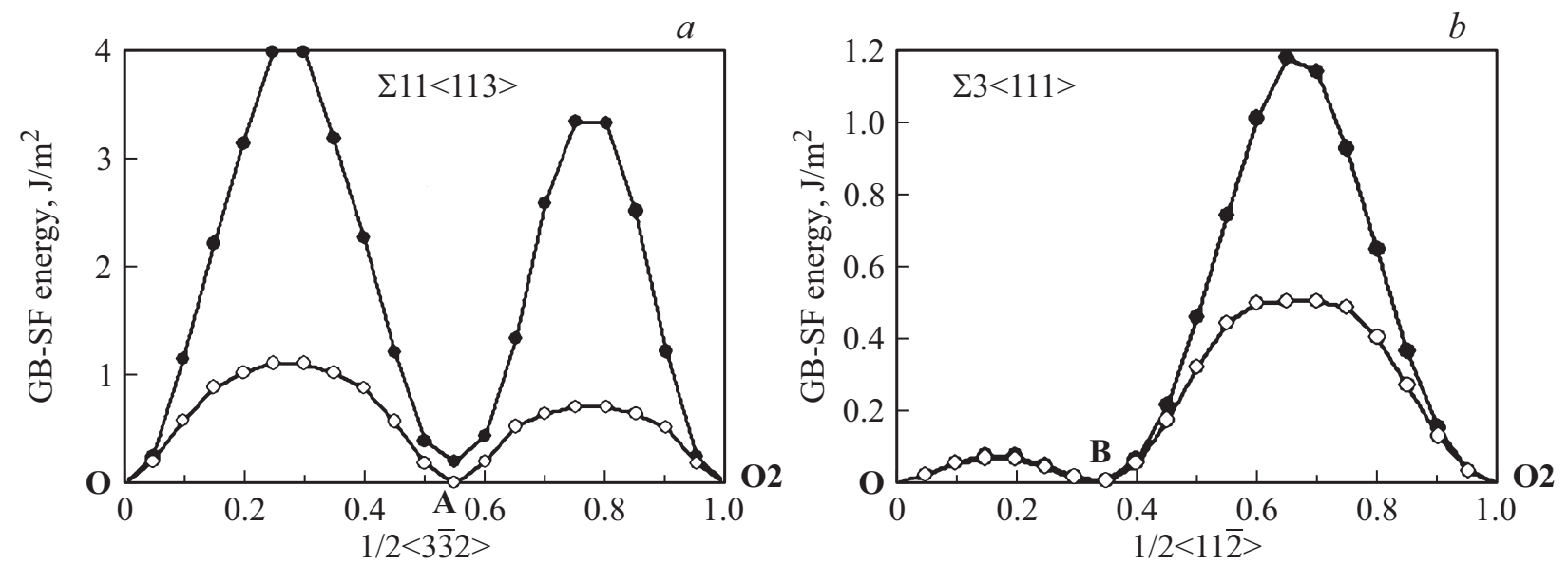

Рис. 6. Зависимость энергии SF вдоль направления, перпендикулярного оси наклона, для границ $\Sigma 11\{113\}\langle 110\rangle(a)$ и $\Sigma 3\{111\}\langle 110\rangle(b)$. Потенциал Pot1.

на GB-SF поверхности присутствует дополнительный минимум в точке А с координатами $(0.55 ; 0.0)$, а также в эквивалентной ей точке A1 с координатами $(0.05 ; 0.5)$.

Рис. $5, b$ свидетельствует о качественно различной зависимости GB-SF после релаксации для сдвига вдоль направления $\langle 110\rangle$ для ГЗ $\Sigma 11\{113\}$ для потенциалов Pot1 (светлые точки на рис. $5, b$ ) и Pot2 (светлые квадраты на рис. $5, b$ ). Для потенциала Pot2 получен локальный минимум при сдвиге вдоль этого направления в точке $1 / 4[110]$. Он не слишком глубокий, но значение $\gamma_{\text {us }}$ для частичного сдвига ниже, чем для полного сдвига на вектор трансляции. Существование частичного сдвига означает, что дислокация с вектором Бюргерса $1 / 2\langle 110\rangle$ вблизи ГЗ может быть расщеплена на 2 частичные в соответствии с реакцией:

$$
1 / 2\langle 110\rangle=1 / 4\langle 110\rangle+\mathrm{SF}+1 / 4\langle 110\rangle .
$$

Расщепление полной дислокации в ГЦК-металлах на две частичные в соответствии с реакцией (1) было предложено в [33], где обсуждались условия реализации подобного расщепления вместо обычного расщеплением на две частичные дислокации Шокли. Было показано, что энергетическая выгодность расщепления (1) зависит от отношения энергии SF, характеризуемой сдвигом $1 / 4\langle 110\rangle$, к энергии SF-вычитания в плоскости $\{111\}$ ГЦК-металла. Для плоскости ГЗ $\Sigma 11\{113\}$ подобные варианты расщепления дислокаций ранее не обсуждались.

В табл. 4 представлены значения энергии нестабильного SF $\gamma_{\text {us, }}$ рассчитанные без релаксации и с учетом релаксации для двух потенциалов (Pot1 и Pot2) в сравнении с результатами расчетов ab initio. Видно, что энергетический барьер для проскальзывания по ГЗ $\Sigma 11\{113\}$ (характеризуемый релаксированной величиной $\left.\gamma_{\text {us }}\right)$ невелик и сравним с аналогичной величиной для Г了 $\Sigma 5$ (см. раздел 3.1). Поэтому следует ожидать, что зернограничное проскальзывание вдоль оси наклона для ГЗ $\Sigma 11\{113\}$ будет облегчено. В тоже время, в отличие
Таблица 4. Энергии нестабильных $\mathrm{SF}\left(\gamma_{\text {us }}, \mathrm{J} / \mathrm{m}^{2}\right)$ для сдвига вдоль направления $\langle 110\rangle$.

\begin{tabular}{c|c|c|c|c}
\hline $\begin{array}{c}\text { Метод } \\
\text { расчета }\end{array}$ & $\begin{array}{c}\Sigma 11\{113\} \\
\text { unrelax }\end{array}$ & $\begin{array}{c}\Sigma 11\{113\} \\
\text { relax }\end{array}$ & $\begin{array}{c}\Sigma 3\{111\} \\
\text { unrelax }\end{array}$ & $\begin{array}{c}\Sigma 3\{111\} \\
\text { relax }\end{array}$ \\
\hline Pot1 & 0.39 & 0.19 & 0.46 & 0.32 \\
Pot2 & 0.22 & 0.14 & 0.38 & 0.27 \\
ab initio & 0.44 & 0.12 & 0.51 & 0.22
\end{tabular}

от ГЗ $\Sigma 5$, проскальзывание в направлении, перпендикулярном оси наклона требует преодоления значительного барьера; в этом случае релаксированная величина $\gamma_{\text {us }} \approx 0.7-1.0 \mathrm{~J} / \mathrm{m}^{2}$ (см. рис. 6,a). Для ГЗ $\Sigma 11\{113\}$ изменение структуры границы после сдвига на вектор О2А приводит к ее эффективному смещению в направлении, перпендикулярном плоскости границы, на величину равную вектору Бюргерса зернограничных сдвигов вдоль направления $\langle 113\rangle\left(\mathbf{b}_{\mathrm{gb}}(\langle 113\rangle)=1 / 11\langle 113\rangle \sim 0.30 a\right)$ и восстановлению идеальной структуры ГЗ, но смещенной относительно стартового состояния.

Для двойниковой ГЗ $\Sigma 3\{111\}\langle 110\rangle$ форма GB-SF поверхности подобна той, которая была получена для ГЗ $\Sigma 5$ (рис. 1). В частности, на ней также имеется дополнительный минимум в точке с координатами $(0.33 ; 0.0)$, который соответствует двойниковому частичному сдвигу ОВ с вектором 1/6 $\langle 112\rangle$, перпендикулярным оси наклона; этому вектору соответствует легкое направление смещения зерен вдоль ГЗ $\Sigma 3\{111\}\langle 110\rangle$ (см. рис. 6, b). Повторение сдвига ОВ в последовательных плоскостях приведет к миграции ГЗ $\Sigma 3\{111\}\langle 110\rangle$ подобно тому, как скольжение двойникующей дислокации в плоскости двойника сопровождается увеличением его толщины.

В табл. 5 представлены энергии нестабильных SF при образовании сдвигов вдоль направления $\mathbf{O O 2}$ для двух рассмотренных ГЗ. Видно, что самые низкие значения $\gamma_{\text {us }}$ реализуются для ГЗ $\Sigma 3\{111\}$. Для ГЗ 
Таблица 5. Энергии нестабильных $\mathrm{SF}\left(\gamma_{\mathrm{us}}, \mathrm{J} / \mathrm{m}^{2}\right)$ для сдвига вдоль вектора направления, перпендикулярного оси наклона $\langle 110\rangle$ границ

\begin{tabular}{c|c|c|c|c}
\hline $\begin{array}{c}\text { Метод } \\
\text { расчета }\end{array}$ & $\begin{array}{c}\Sigma 11\{113\}, \\
\text { сдвиг О2A } \\
\text { unrelax }\end{array}$ & $\begin{array}{c}\Sigma 11\{113\}, \\
\text { сдвиг О2A } \\
\text { relax }\end{array}$ & $\begin{array}{c}\Sigma 3\{111\}, \\
\text { сдвиг ОВ } \\
\text { unrelax }\end{array}$ & $\begin{array}{c}\Sigma 3\{111\}, \\
\text { сдвиг ОВ } \\
\text { relax }\end{array}$ \\
\hline Pot1 & 3.3 & 0.70 & 0.09 & 0.08 \\
Pot2 & 2.7 & 0.67 & 0.14 & 0.13 \\
ab initio & 1.9 & 0.71 & 0.09 & 0.08
\end{tabular}

$\Sigma 11\{113\}$, напротив, энергия нестабильных ДУ очень высока, значительно выше, чем для сдвига вдоль направления $\langle 110\rangle$ в этой плоскости. Таким образом, для границы $\Sigma 11\{113\}$ наиболее предпочтительным является сдвиг вдоль направления $\langle 110\rangle$, параллельного оси наклона. Для ГЗ $\Sigma 3\{111\}$ возможен частичный сдвиг $1 / 6\langle 112\rangle$, перпендикулярный оси наклона; повторение таких сдвигов в соседних плоскостях будет сопровождаться миграцией двойниковой границы. Альтернативным механизмом проскальзывания по ГЗ $\Sigma 3\{111\}$ может быть смещение зерен вдоль составного пути OB-O3 подобного тому, который обсуждался выше для ГЗ $\Sigma 5\{012\}\langle 100\rangle$. Такое смещение зерен по плоскости $\{111\}$ соответствует последовательному движению частичных дислокаций Шокли.

\section{4. Обсуждение результатов}

С целью выяснения механизмов, контролирующих подвижность ГЗ в поликристалле $\mathrm{Al}$, методами атомистического моделирования исследована структура ГЗ и энергетические характеристики процесса проскальзывания для ряда типичных специальных ГЗ наклона с $\Sigma=3,5,11$. Расчет энергии зернограничного обобщенного дефекта упаковки $(\mathrm{GB}-\mathrm{SF})$ позволил дать оценку величины барьера, определяющего сопротивление относительному смещению зерен и установить наиболее легкие направления для зернограничного проскальзывания. Значения энергий $\mathrm{GB}-\mathrm{SF}$, полученные методом молекулярной динамики, находятся в разумном согласии с результатами PAW-VASP расчетов ab initio, что позволяет говорить о надежности полученных результатов. Показано, что для рассмотренных ГЗ энергетически выгодным является реализация частичного сдвига соседних зерен (величина которого меньше трансляции РСУ); при этом такой сдвиг сопровождается консервативной миграцией ГЗ.

Наиболее низкое значение $\gamma_{\text {us }}=0.05 \mathrm{~J} / \mathrm{m}^{2}$ (ab initio расчет) получено для ГЗ $\Sigma 5\{013\}$ (табл. 3) для частичного сдвига ОВ вдоль направления $\langle 013\rangle$, перпендикулярного оси наклона. Значения энергий $\gamma_{\text {us }}$ в плоскости $\{111\}\left(0.08 \mathrm{~J} / \mathrm{m}^{2}\right.$, табл. 5) близки к значениям $\gamma_{\text {us }}$ для плоскости $\{113\}\left(0.12 \mathrm{~J} / \mathrm{m}^{2}\right.$, табл. 4) и составляет
$0.20 \mathrm{~J} / \mathrm{m}^{2}$ (табл. 3) для самой низкоэнергетической границы $\Sigma 5\{012\}$. Другие сдвиги для рассмотренных ГЗ приводят к существенно более высоким значениям $\gamma_{\text {us }}$, в пределах $0.30-0.70=\mathrm{J} / \mathrm{m}^{2}$.

Оценка напряжения, необходимого для проскальзывания путем относительного смещения зерен как целого (теоретический предел прочности на сдвиг) дает $\sigma_{s} \sim 2 \pi \gamma_{\text {us }} / \mathbf{b}$, где $b-$ вектор сдвига. Для легких сдвигов величина $\sigma_{s}$ весьма мала и лежит в пределах 30-120 МПа, что не превышает предел текучести $\mathrm{Al}$ $\sigma_{y}$. Для остальных рассмотренных сдвигов оценка дает большие значения $\sigma_{s}>\sigma_{y}$; в этом случае относительное смещение зерен по ГЗ будет осуществляется движением зернограничных дислокаций.

Наиболее простым вариантом зернограничного скольжения, при котором для каждого сдвига структура границы восстанавливается, является сдвиг на вектор Бюргерса $1 / 2\langle 110\rangle$, который совпадает с решеточным сдвигом, в плоскости $\{113\}$ для симметричной ГЗ $\Sigma 11\{113\}\langle 110\rangle$. Для остальных вариантов с низкими значениями $\gamma_{\text {us }}$ зернограничное скольжение сопровождается смещением границы в направлении перпендикулярном к плоскости ГЗ на расстояние, кратное вектору Бюргерса зернограничных дислокаций (ЗГД). Многократное скольжение ЗГД по системе последовательных параллельных плоскостей должно приводить к изменению размера зерна. В табл. 6 приведены значения векторов сдвига нормальных $m$ и параллельных s плоскости ГЗ, а также параметр взаимного влияния процессов миграции и скольжения $\beta=s / m[14,15]$. Видно, что параметр $\beta$ может изменяться в широких пределах, достигая наибольшего значения для $\Sigma 5\{012\}$..

Таким образом, полученные результаты показывают, что процесс проскальзывания по ГЗ имеет сильную ориентационную зависимость; кроме легких направлений сдвига имеются также и тяжелые, вдоль которых проскальзывание вряд ли возможно. Кроме того, мы обнаружили, что многие легкие процессы проскальзывания включают частичный сдвиг, реализация которого сопровождается консервативной миграцией ГЗ. Поэтому они могут играть существенную роль в деформации поликристалла при пониженных температурах, когда контролируемые диффузией процессы релаксации заморожены.

Следует отметить, что рассмотренные выше процессы миграции ГЗ, инициированные частичным зерногранич-

Таблица 6. Нормальные $m$ и параллельные $s$ плоскостям ГЗ векторы сдвига и параметры взаимного влияния $\beta$

\begin{tabular}{c|c|c|c}
\hline Границы зерен & $m$ & $s$ & $\beta$ \\
\hline$\Sigma 5\{013\}$ & $0.05\langle 013\rangle$ & $0.1\langle 013\rangle$ & 2.0 \\
$\Sigma 5\{012\}$ & $0.1\langle 012\rangle$ & $0.4\langle 012\rangle$ & 4.0 \\
$\Sigma 11\{113\}$ & $0.30\langle 113\rangle$ & $-0.225\langle 332\rangle$ & 1.06 \\
$\Sigma 3\{111\}$ & $0.333\langle 11\rangle$ & $0.167\langle 112\rangle$ & 0.71
\end{tabular}


ным сдвигом реализуются для специфического случая симметричных специальных ГЗ наклона. В мелкозернистом поликристалле с преобладанием произвольных ГЗ механизм индуцированной напряжением миграции границ может быть более сложным и включать протекающие на мезоуровне процессы, обеспечивающие проскальзывание несимметричных ГЗ путем движения уступов [11] и совместность деформации в тройных стыках зерен [9]. Тем не менее, полученные в настоящей работе результаты дают представление о фундаментальных механизмах, определяющих проскальзывание зерен и условиях реализации инициированного напряжением процесса миграции ГЗ.

\section{5. Заключение}

Методами атомистического моделирования исследована структура ГЗ и энергетические характеристики процесса проскальзывания для ряда типичных специальных ГЗ наклона $\Sigma 5(012), \Sigma 5(013), \Sigma 11(113)$ и $\Sigma 3(111)$. Дана оценка величины барьера, определяющего сопротивление относительному смещению зерен, и определены наиболее легкие направления для зернограничного проскальзывания, для которых необходимое критическое напряжение весьма мало и не превышает предел текучести. Показано, что для рассмотренных ГЗ энергетически выгодным является реализация частичного сдвига соседних зерен, который сопровождается консервативной миграцией ГЗ. Процессы миграции ГЗ, инициированные частичным зернограничным сдвигом могут играть существенную роль в низкотемпературной деформации мелкозеренных поликристаллов $\mathrm{Al}$.

\section{Список литературы}

[1] M. Guillope, J.P. Poirier. Acta Met. 28, 163 (1980)

[2] S.E. Babcock, R.W. Balluffi. Acta Met. 37, 2357;2367 (1989).

[3] K.W. Schiotz, K.W. Jacobsen. Science 301, 1357 (2003).

[4] H. Van Swygenhoven, J.R. Weertman. Mater. Today 9, 24 (2006).

[5] D. Farkas, A. Froseth, H. Van Swygenhoven. Scripta Mater. 55, 695 (2006).

[6] D.S. Gianola, S. Van Petegem, M. Legros, S. Brandstetter, H. Van Swygenhoven, K.J. Hemker. Acta Mater. 54, 2253 (2006).

[7] M. Legros, D.S. Gianola, K.J. Hember. Acta Mater. 56, 3380 (2008).

[8] J.W. Cahn, Yu. Mishin, A. Suzuki. Acta Mater. 54, 4953 (2006).

[9] М.Ю. Гуткин, К.Н. Микаедян, И.А. Овидько. ФТТ 50, 1266 (2008).

[10] S.V. Bobylev, N.F. Morozov, I.A. Ovid'ko. Phys. Rev. B 84, 094103 (2011).

[11] R. Hadian, B. Grabowski, C.P. Race, J. Neugebauer. Phys. Rev. B 94, 165413 (2016).

[12] T.G. Langdon. J. Mater. Sci. 41, 597 (2006).

[13] J. Li, A.K. Soh. Acta Mater. 61, 5449 (2013).
[14] F. Mompiou, D. Caillard, M. Legros. Acta Mater. 57, 2198; 2390 (2009).

[15] F. Mompiou, M. Legros, D. Caillard. Acta Mater. 58, 3676 (2010).

[16] L. Lu, X. Chen, X. Huang, K. Lu. Science 323, 607 (2009).

[17] G. Purceka, O. Saraya, M.I. Nagimovb, A.A. Nazarov, I.M. Safarov, V.N. Danilenko, O.R. Valiakhmetov, R.R. Mulyukov. Phil. Mag. 92, 690 (2012).

[18] Y.F. Shen, L. Lu, Q.H. Lu, Z.H. Jin, K. Lu. Scripta Mater. 52, 989 (2005).

[19] V. Vitek. Cryst. Latt. Def. 5, 1 (1974).

[20] O.N. Mryasov, Yu.N. Gornostyrev, van M. Schilfgaarde, A.J. Freeman. Mater. Sci. Eng. A 138, 309 (2001).

[21] M.A. Tschopp, D.L. Macdowell. Phil. Mag. 87, 3871 (2007).

[22] http://lammps.sandia.gov/index.html

[23] F. Apostol, Y. Mishin. Phys. Rev. B 83, 054116 (2011).

[24] M.I. Mendelev, M. Asta, M.J. Rahman, J.J. Hoyt. Phil. Mag. 89, 3269 (2009).

[25] http://www.ctcms.nist.gov/potentials/

[26] G. Kresse, J. Furthmüller. Phys. Rev. B 54, 11169 (1996).

[27] G. Kresse, J. Hafner. J. Phys.: Condens. Matter 6, 8245 (1994).

[28] G. Kresse, D. Joubert. Phys. Rev. B 59, 1758 (1999).

[28] J.P. Perdew, J.A. Chevary, S.H. Vosko, K.A. Jackson, M.R. Pederson, D.J. Singh, C. Fiolhais. Phys. Rev. B 46, 6671 (1992).

[30] S.H. Vosko, L. Wilk, M. Nusair. Can. J. Phys. 58, 1200 (1980).

[31] A. Kelly, W. Tyson, A.H. Cottrell. Phil. Mag. 15, 567 (1967).

[32] W. Bollmann. Crystal defects and crystalline interfaces. Berlin: Springer-Verlag, 1970, $254 \mathrm{p}$.

[33] V. Vitek \& F. Kroupa. Phil. Mag. A 19, 265 (1969).

Редактор Ю.Э. Китаев 